\title{
A prática do aleitamento materno em crianças com fissuras labiopalatinas
}

\author{
The practice of breastfeeding in children with labiopalatinal fissures
}

La práctica del alcance materno en niños con fisuras labiopalatinas

\begin{abstract}
Sabrina Maria Ribeiro Amorim*, Marianne Rocha Duarte de Carvalho1, Aziz Moisés Alves da Costa $^{1}$, Ravena de Sousa Alencar Ferreira' ${ }^{1}$, Teresa Amélia Carvalho de Oliveira ${ }^{1}$, Vitor Kauê de Melo Alves ${ }^{1}$, Whesley Fenesson Alves dos Santos ${ }^{1}$, Daiane da Silva Azevedo ${ }^{1}$, Erlane Pereira da Silva ${ }^{1,2}$, Elayne Maria Magalhães ${ }^{3}$, Mariana Alves Farias ${ }^{4}$.
\end{abstract}

\section{RESUMO}

Objetivo: Avaliar a prática do aleitamento materno em crianças com fissuras labiopalatinas atendidas em um serviço de referência no estado do Piauí. Método: Trata-se de um estudo epidemiológico, descritivo com abordagem quantitativa cuja amostra foi constituída por 25 mães de crianças com fissuras labiopalatinas atendidas em um hospital de referência. Os dados foram coletados mediante aplicação do questionário semiestruturado, e analisados estatisticamente utilizando-se o software Stastistical Package for the Social Sciences, versão 21.0. Resultados: Constatou-se predominância de crianças do sexo masculino, provenientes do interior do estado e nascidos em hospital público. A fissura pré-forame incisivo apresentou maior incidência. A maior parte das mães recebeu algum tipo de orientação acerca do aleitamento materno no período gestacional e o profissional enfermeiro foi o que mais orientou. Tanto no ambiente hospitalar quanto domiciliar, prevaleceu o aleitamento materno oferecido na mamadeira e as crianças que tiveram uma pega efetiva no seio possuíam fissura pré e transforame incisivo. Conclusão: As orientações sobre o aleitamento requerem preparo e conhecimento dos profissionais de saúde acerca das técnicas e posicionamentos corretos para a amamentação do fissurado e a falta dela, pode influenciar negativamente 0 processo e contribuir para o desmame precoce.

Palavras-chave: Fissura palatina, Fenda labial, Aleitamento materno.

\begin{abstract}
Objective: To evaluate the practice of breastfeeding in children with cleft lip and palate treated at a referral service in the state of Piauí. Method: This is an epidemiological, descriptive study with a quantitative approach whose sample consisted of 25 mothers of children with cleft lip and palate treated at a referral hospital. The data were collected using the semi-structured questionnaire, and analyzed statistically using the software Stastistical Package for the Social Sciences, version 21.0. Results: It was found predominance of male children, from the interior of the state and born in a public hospital. The incisor pre-foramen cleft had a higher incidence. Most mothers received some guidance about breastfeeding during the gestational period and the nurse practitioner was the one who most advised them. Both in the hospital and in the home environment, breastfeeding prevailed in the bottle and the children who had an effective grasp in the sinus had pre-cleft and incisor transforaments. Conclusion: The guidelines on breastfeeding require training and knowledge of health professionals about correct techniques and positioning for breastfeeding and lack of it, may negatively influence the process and contribute to early weaning.
\end{abstract}

Key words: Cleft palate, Cleft Lip, Breast feeding.

\footnotetext{
1 Universidade Estadual do Piauí (UESPI), Teresina - PI. E-mail: sabrina002amorim@gmail.com

2 Hospital São Paulo (HSP), Teresina - PI.

${ }^{3}$ Faculdade do Piauí (FAPI), Teresina - PI.

${ }^{4}$ Faculdade Estácio de Teresina (CEUT). Teresina - PI.
} 


\section{RESUMEN}

Objetivo: Evaluar la práctica de la lactancia materna en niños con fisuras labiopalatinas atendidas en un servicio de referencia en el estado de Piauí. Método: Se trata de un estudio epidemiológico, descriptivo con abordaje cuantitativo cuya muestra fue constituida por 25 madres de niños con fisuras labiopalatinas atendidas en un hospital de referencia. Los datos fueron recolectados mediante la aplicación del cuestionario semiestructurado, y analizados estadísticamente utilizando el software Stastistical Package for the Social Sciences, versión 21.0. Resultados: Se constató predominancia de niños del sexo masculino, provenientes del interior del estado y nacidos en un hospital público. La fisura prefeta incisiva presentó mayor incidencia. La mayoría de las madres recibieron algún tipo de orientación sobre la lactancia materna en el período gestacional y el profesional enfermero fue el que más orientó. Tanto en el ambiente hospitalario cuanto domiciliar, prevaleció la lactancia materna ofrecida en el biberón y los niños que tuvieron una asa efectiva en el seno poseían fisura pre y transforme incisivo. Conclusión: Las orientaciones sobre la lactancia requieren preparación y conocimiento de los profesionales de la salud acerca de las técnicas y posicionamientos correctos para la lactancia del fisurado y la falta de ella, puede influenciar negativamente el proceso y contribuir al destete precoz.

Palabras clave: Fisura palatina, Fenda Labial, Lactancia materna.

\section{INTRODUÇÃO}

O aleitamento materno se dá quando a criança recebe o leite materno direto da mama ou ordenhado, ou leite humano de outra fonte, sem outros líquidos ou sólidos, com exceção de gotas ou xaropes contendo vitaminas, sais de reidratação oral, suplementos minerais ou medicamento (BRASIL, 2009).

A Organização Mundial da Saúde (OMS) e o Ministério da Saúde (MS) recomendam aleitamento materno exclusivo por seis meses e complementado até os dois anos ou mais e considera o melhor alimento para o bebê tendo em vista seus inúmeros benefícios uma vez que possui nutrientes, fatores de proteção para o lactente contra infecções e fatores que favorecem o seu crescimento e o desenvolvimento do sistema imunológico (BRASIL, 2009).

Alguns fatores podem interferir na prática do aleitamento materno, tornando-a difícil ou trazendo complicações. Dentre estes fatores podem-se destacar os problemas relacionados as mamas, a pega incorreta e as situações especiais como a presença de má formações orofaciais, dentre as quais de destaca as fissuras labiopalatinas (FLP).

As fissuras labiopalatinas são malformações congênitas que ocorrem entre a $4^{\mathrm{a}}$ e $12^{\mathrm{a}}$ semana do período embrionário, devido à falta de fusão dos processos maxilar e médio-nasal (CARVALHO LRRA, 2010). São as mais freqüentes malformações faciais e, no Brasil, estima- se a ocorrência da ordem de 1 para cada 650 nascimentos (WEHBY GL e CASSELL CH, 2010). Estas podem ser classificadas utilizando-se como ponto de referência o forame incisivo, limite entre palato primário (pró-lábio, pré-maxila e septo cartilaginoso) e o secundário, o que permite a diferenciação em três tipos principais de fissuras: fissura pré-forame incisivo, fissura pós-forame incisivo e fissura transforame incisivo, considerada de maior gravidade, que pode ocorrer de forma unilateral ou bilateral, atingindo lábio, rebordo alveolar e todo palato (SPINA V et al., 1972).

A alimentação do fissurado labiopalatino é, na maioria das vezes, muito difícil, ainda mais quando a família não recebe orientação. A dificuldade de alimentação de bebês com fissura surge logo após o nascimento, devido ao prejuízo no mecanismo de sucção e deglutição, decorrente da falta de integridade das estruturas anatômicas (DI NINNO et al., 2011). Por isso, as principais dificuldades relatados pelas mães de crianças com FLP são a asfixia, o refluxo nasal e dificuldade de sucção (FIERRO CM e SALAZAR ES et al., 2013).

Diante do exposto, o presente estudo objetivou avaliar a prática do aleitamento materno em crianças com fissuras labiopalatinas atendidas em um serviço de referência. 


\section{MÉTODOS}

Trata-se de um estudo epidemiológico, descritivo com abordagem quantitativa realizado em um centro de referência em atendimento de alta complexidade a pacientes portadores de anomalias craniofaciais no Piauí. A amostra do estudo foi constituída por 25 mães de crianças com FLP, na faixa etária de 0 a 5 anos de idade, de ambos os gêneros, independente do tipo de fissura que apresentavam. As mães foram identificadas durante $o$ atendimento ambulatorial do serviço do Hospital de referência num período compreendido entre junho e julho de 2017. Foram excluídas mães de crianças com Síndrome de Pierre Robin que apresentavam comprometimento do Sistema Nervoso Central e outras anomalias congênitas associadas ou sindrômicas.

Para levantamento dos dados utilizou-se um questionário semi-estruturado criado por Thomé $S$ (1990) e modificado por Silva et al. (2005), que avaliava as dificuldades do aleitamento das crianças e os métodos utilizados nos primeiros meses de vida. Ressalta-se que o instrumento de coleta foi aplicado de forma individualizada a cada uma das mães que aceitaram participar do estudo após o esclarecimento dos objetivos da pesquisa e assinatura em duas vias do Termo de Consentimento Livre e Esclarecido.

Após a coleta, compôs-se um banco de dados, que foram processados no programa Stastistical Package For The Social Sciences SPSS $\AA$, versão 21.0 para Windows ${ }^{\circledR}$. Foi realizada a análise descritiva dos dados, apresentando valores absolutos e porcentagens, média e desvio padrão e analisados descritivamente medindo-se a taxa de associação entre as variáveis do estudo, sendo os resultados apresentados sob a forma de tabelas e gráficos. Estes por sua vez, foram confrontados com a literatura existente com o intuito de levantar discussões e de se reiterar a veracidade dos dados em análise.

Em relação aos aspectos éticos, a pesquisa seguiu as recomendações da Resolução № 466 de 2012 do Conselho Nacional de Saúde (CNS), tendo sido apreciada e autorizada pelo Comitê de Ética em Pesquisa da Universidade Estadual do Piauí (CEP-UESPI) conforme CAAE 68075717.9.3001.5584 e parecer no 2.134.377.

\section{RESULTADOS}

Após o levantamento de dados, constatou-se que o perfil das crianças com fissuras labiopalatais atendidas no hospital cenário deste estudo mostra maior predominância de crianças do sexo masculino $(56 \%)$, nascidos em hospital publico (92\%) e provenientes do interior do estado (52\%). No que concerne ao tipo de fissura, utilizando a classificação de Spina et al. (1972), constatou-se maior ocorrência de crianças com fissuras do tipo pré-forame incisivo (64\%), sendo a fissura pré-forame incisivo bilateral a de maior incidência representando $36 \%$ dos casos (Tabela 1).

Tabela 1- Perfil das crianças com fissuras labiopalatinas atendidas no serviço de referência. Teresina, 2017.

\begin{tabular}{llcc}
\hline & \multicolumn{1}{c}{ Varável } & $\mathbf{n}$ & $\%$ \\
\hline \multirow{2}{*}{ Gênero } & Masculino & 14 & 56,0 \\
& Feminino & 11 & 44,0 \\
\hline \multirow{2}{*}{ Hospital de nascimento } & Público & 23 & 92,0 \\
& Privado & 2 & 8,0 \\
\hline \multirow{2}{*}{ Procedência } & Capital & 7 & 28,0 \\
& Interior do estado & 13 & 52,0 \\
& Outros estados & 5 & 20,0 \\
\hline \multirow{2}{*}{ Tipo de fissura } & Fissura pré-forame incisivo unilateral direita completa & 1 & 4,0 \\
(Classificação de & Fissura pré-forame incisivo unilateral direita incompleta & 6 & 24,0 \\
Spina et al., 1972) & Fissura pré-forame incisivo bilateral & 9 & 36,0 \\
& Fissura pós-forame incisivo completa & 3 & 12 \\
& Fissura transforame incisivo & 6 & 24 \\
\hline \multicolumn{1}{c}{ Total } & & $\mathbf{2 5}$ & $\mathbf{1 0 0 , 0}$ \\
\hline
\end{tabular}

Fonte: Dados da Pesquisa. 
Em relação as informações recebidas pelas mães acerca da presença de fissuras das crianças no período gestacional da criança, a grande maioria (96\%) relatou desconhecer a presença da fissura antes do nascimento. Quanto às orientações a cerca do aleitamento materno recebidas antes do nascimento, $84 \%$ das mães informaram ter recebido algum tipo de orientação, sendo o profissional enfermeiro (84\%) aquele que mais orientou. No que se refere ao tipo de informação recebida, $84 \%$ das mães afirmaram ter recebido informações relacionadas à importância da prática do aleitamento materno.

Após o nascimento da criança, a maioria das mães (92\%) viu a criança logo após o nascimento, e a maior parte delas $(60 \%)$ reagiu de maneira assustada e/ou chorou diante da situação vivenciada. O profissional responsável por informar à mãe sobre a presença da malformação no bebê foi o médico, em $84 \%$ dos casos, seguido pelo enfermeiro (12\%) (Tabela 2).

Tabela 2 - Caracterização das informações recebidas pelas mães das crianças fissuradas no período gestacional e após o parto. Teresina, 2017.

\begin{tabular}{|c|c|c|c|}
\hline \multicolumn{2}{|c|}{ Informações sobre fissurados antes do nascimento } & \multirow{2}{*}{$\begin{array}{c}\mathbf{n} \\
24 \\
1\end{array}$} & \multirow{2}{*}{$\begin{array}{c}\% \\
96,0 \\
4,0\end{array}$} \\
\hline $\begin{array}{l}\text { Soube da presença de fissuras antes } \\
\text { do nascimento }\end{array}$ & $\begin{array}{l}\text { Não } \\
\text { Sim }\end{array}$ & & \\
\hline \multirow{2}{*}{$\begin{array}{l}\text { Orientação do aleitamento antes do } \\
\text { nascimento }\end{array}$} & Não & 4 & 16,0 \\
\hline & Sim & 21 & 84,0 \\
\hline Quem orientou & $\begin{array}{l}\text { Médico } \\
\text { Enfermeiro } \\
\text { Sem orientação }\end{array}$ & $\begin{array}{c}8 \\
13 \\
4\end{array}$ & $\begin{array}{l}32,0 \\
52,0 \\
16,0\end{array}$ \\
\hline \multirow[t]{2}{*}{ Quais orientações } & $\begin{array}{l}\text { Relacionadas a importância do aleitamento } \\
\text { materno }\end{array}$ & 21 & 84,0 \\
\hline & Sem orientação & 4 & 16,0 \\
\hline Viu o bebê logo que nasceu & $\begin{array}{l}\text { Sim } \\
\text { Não } \\
\text { ficou assustada / chorou }\end{array}$ & $\begin{array}{c}23 \\
2 \\
5\end{array}$ & $\begin{array}{c}92,0 \\
8,0 \\
60,0\end{array}$ \\
\hline \multirow[b]{2}{*}{ Reação ao problema do bebê } & não aceitou & 5 & 20,0 \\
\hline & $\begin{array}{l}\text { aceitou } \\
\text { ficou preocupada }\end{array}$ & $\begin{array}{l}3 \\
2\end{array}$ & $\begin{array}{l}12,0 \\
8,0\end{array}$ \\
\hline Profissional que deu a noticia & $\begin{array}{l}\text { Médico } \\
\text { Enfermeiro }\end{array}$ & $\begin{array}{c}21 \\
3\end{array}$ & $\begin{array}{l}84,0 \\
12,0\end{array}$ \\
\hline \multirow{2}{*}{$\begin{array}{l}\text { Informação que recebeu sobre a } \\
\text { fissura }\end{array}$} & $\begin{array}{l}\text { Sem informação } \\
\text { Tem tratamento }\end{array}$ & $\begin{array}{c}1 \\
21\end{array}$ & $\begin{array}{c}4,0 \\
84,0\end{array}$ \\
\hline & Nenhuma & 4 & 16,0 \\
\hline Total & & 25 & 100,0 \\
\hline
\end{tabular}

Fonte: Dados da Pesquisa.

Quanto a caracterização da prática do aleitamento materno em crianças com fissuras labiopalatinas durante o período de permanência hospitalar, independentemente do método utilizado, observou-se que 24 crianças $(96 \%)$ foram alimentadas com leite materno, com ou sem complementação. As mães afirmaram que entre as crianças com fissuras labiopalatinas, $60 \%$ delas tiveram dificuldades com a pega da criança, mas que, no entanto, $36 \%$ destas crianças mamaram no peito. Aquelas que apresentaram dificuldade para oferecer o peito e optaram pelo uso da mamadeira, referiram fazer a ordenha manual do leite materno antes de oferecê-lo à criança (40\%) (Tabela 3). 
Tabela 3 - A prática do aleitamento materno em crianças com fissuras labiopalatinas no período hospitalar e primeiros seis meses de vida. Teresina, 2017.

\begin{tabular}{|c|c|c|c|}
\hline \multicolumn{2}{|c|}{ Aleitamento materno dos fissurados após o nascimento } & \multirow{2}{*}{$\begin{array}{c}\mathbf{n} \\
25\end{array}$} & \multirow{2}{*}{\begin{tabular}{c|}
$\%$ \\
100,0
\end{tabular}} \\
\hline Tontou amamentar no coin & Sim & & \\
\hline 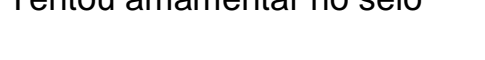 & Não & 0 & 0,0 \\
\hline \multirow{2}{*}{ Criança pegou o seio } & Sim & 10 & 40,0 \\
\hline & Não & 15 & 60,0 \\
\hline \multirow{2}{*}{$\begin{array}{l}\text { Leite do peito alguma vez nos } \\
\text { primeiro } 6 \text { meses }\end{array}$} & Sim & 24 & 96,0 \\
\hline & Não & 1 & 4,0 \\
\hline \multirow{3}{*}{ Motivo } & No hospital falaram para dar leite de peito & 21 & 84,0 \\
\hline & Já havia dado leite de peito para outros filhos & 3 & 12,0 \\
\hline & Sem informação & 1 & 4,0 \\
\hline \multirow{4}{*}{ Como } & Peito & 9 & 36,0 \\
\hline & Mamadeira & 9 & 36,0 \\
\hline & Outros & 3 & 12,0 \\
\hline & Sem informação & 1 & 4,0 \\
\hline \multirow{3}{*}{ Como retiro o leite } & Com bombinha & 6 & 24,0 \\
\hline & Manualmente & 10 & 40,0 \\
\hline & Não retirou o leite & 9 & 36,0 \\
\hline Total & & 25 & 100,0 \\
\hline
\end{tabular}

Fonte: Dados da Pesquisa.

Com relação ao tipo de aleitamento das crianças com fissuras labiopalatinas, notou-se que no ambiente hospitalar prevaleceu o aleitamento materno oferecido na mamadeira $(48 \%)$ e apenas $8 \%$ das crianças utilizaram algum tipo de fórmula artificial. No ambiente domiciliar $68 \%$ das crianças foram alimentadas com leite materno sendo a mamadeira o método mais utilizado (60\%), $24 \%$ associaram o leite natural materno e outro tipo de leite artificial e $8 \%$ utilizaram exclusivamente leite artificial (Tabela 4).

No que se refere à posição mais empregada pela mãe para amamentar seu filho, $36 \%$ delas relataram a posição sentada como a melhor para a mãe amamentar e $24 \%$ informaram ser essa mesma posição a mais adequada para a criança mamar. A maioria, no entanto relatou não existir uma melhor posição para amamentar (48\%) e nem para a criança mamar $(60 \%)$, visto que a maioria das crianças $(60 \%)$ não teve uma pega efetiva ao seio como já demonstrado anteriormente na Tabela 3.

Dentre as crianças que tiveram uma pega efetiva no seio segundo à mãe, 9 delas possuíam fissura préforame incisivo e 1 transforame. 15 (quinze) crianças com fissuras labiopalatinas (60\%) ao serem colocadas no peito, não obtiveram êxito no aleitamento materno, 7 (sete) dessas crianças possuíam fissura pré-forame, 3 (três) pós-forame e 5 (cinco) fissura transforame incisivo (Tabela 5). 
Tabela 4- Métodos e técnicas utilizadas no aleitamento de crianças com fissura labiopalatina. Teresina, 2017.

\begin{tabular}{|c|c|c|c|}
\hline \multicolumn{2}{|c|}{ Métodos e técnicas utilizadas no aleitamento do fissurado } & \multirow{2}{*}{$\begin{array}{l}\mathbf{N} \\
8\end{array}$} & \multirow{2}{*}{$\begin{array}{c}\% \\
32,0\end{array}$} \\
\hline \multirow{5}{*}{$\begin{array}{l}\text { Tipo de aleitamento } \\
\text { na fase hospitalar }\end{array}$} & Exclusivamente ao seio & & \\
\hline & Apenas leite materno na mamadeira & 12 & 48,0 \\
\hline & Leite materno no seio e na mamadeira & 1 & 4,0 \\
\hline & Outro leite & 2 & 8,0 \\
\hline & Outros & 2 & 8,0 \\
\hline \multirow{3}{*}{$\begin{array}{l}\text { Tipo de aleitamento } \\
\text { no domicilio }\end{array}$} & Leite de peito & 17 & 68,0 \\
\hline & Leite de peito e outro tipo de leite & 6 & 24,0 \\
\hline & Apenas outro tipo de leite & 2 & 8,0 \\
\hline \multirow{5}{*}{$\begin{array}{l}\text { Método de } \\
\text { amamentação } \\
\text { domiciliar }\end{array}$} & no peito & 7 & 28,0 \\
\hline & no peito e na mamadeira & 1 & 4,0 \\
\hline & Mamadeira & 15 & 60,0 \\
\hline & Com colher & 1 & 4,0 \\
\hline & Outros & 1 & 4,0 \\
\hline \multirow{4}{*}{$\begin{array}{l}\text { Melhor posição para } \\
\text { Amamentar }\end{array}$} & Sentada & 9 & 36,0 \\
\hline & Deitada & 3 & 12,0 \\
\hline & Nenhuma & 12 & 48,0 \\
\hline & Sem informação & 1 & 4,0 \\
\hline \multirow{4}{*}{$\begin{array}{l}\text { Melhor posição para } \\
\text { Mamar }\end{array}$} & Deitado junto ao corpo da mãe & 3 & 12,0 \\
\hline & Sentado & 6 & 24,0 \\
\hline & Cavalinho & 1 & 4,0 \\
\hline & Nenhuma & 15 & 60,0 \\
\hline
\end{tabular}

Fonte: Dados da Pesquisa.

Tabela 5 - Relação entre tipo de fissura e aleitamento natural ao seio avaliadas em crianças com fissuras labiopalatinas. Teresina, 2017.

\begin{tabular}{cccc}
\hline Variável & \multicolumn{2}{c}{ Notal } & \\
\hline Tipo de fissura & \multicolumn{2}{c}{ Pega efetiva no seio } & \\
\cline { 2 - 4 } & sim & não & 16 \\
Pré forame incisivo & 9 & 7 & 3 \\
Pós forame incisivo & 0 & 3 & 6 \\
Transforame incisivo & 1 & $\mathbf{1 5}$ & $\mathbf{2 5}$ \\
\hline Total & $\mathbf{1 0}$ & & \\
\hline
\end{tabular}

Fonte: Dados da Pesquisa. 


\section{DISCUSSÃO}

A amostra deste estudo apresentou-se homogênea quanto ao gênero, pois dentre as 25 mães de filhos com fissuras labiopalatinas, $56 \%$ deles eram no sexo masculino. Esse dado corrobora com o estudo realizado por Silva RCC et al. (2013) em Sobral (CE), no período de junho de 2009 a março de 2010 onde ficou evidenciado que dos 103 sujeitos pesquisados nascidos com fissura, 61 (59\%) eram do gênero masculino. Esta incidência aumentada da fissura em homens foi também evidenciada no estudo de Baroneza JE et al. (2005) que mostrou uma razão entre os gêneros encontrados de 1,5 masculino:1,0 feminino em pesquisa realizada em Londrina, Paraná.

Quanto à procedência das crianças, foi observado que ocorre o predomínio dos sujeitos nascidos em hospital público (92\%) e provenientes do interior do estado 13 (52\%). Outros estudos também evidenciam esse achado como o de Freitas MCA e Batista TS et al. (2013) que avaliaram a distribuição da fissura labiopalatina no estado da Bahia entre 2000 e 2010 e salientaram que os municípios com maior prevalência foram os do interior.

Em relação ao tipo de fissura, houve neste estudo predomínio das fissuras do tipo pré-forame incisivo (64\%) o que está de acordo com estudo semelhante realizado por Silva et al. (2013) no qual foi possível observar no diagnóstico da cirurgia que as fissuras labiais (pré-forame incisivo) incidiram com maior frequência (44\%). Os dados aqui encontrados divergem da maioria dos estudos revisados que apontaram um maior acometimento da fissura transforame incisivo entre as crianças (CYMROT M et al., 2010; FREITAS JAS et al., 2011; GARDENAL $M$ et al., 2011)

Apesar de ser uma anomalia de diagnóstico possível durante a gestação, a partir da décima quarta semana, através do exame de ultrassom conforme Vaccari-mazzetti, Kobata e Brock (2009), a grande maioria das mães (96\%) que participaram desse estudo relatou não saber da presença da fissura antes do nascimento da criança. Tal fato pode esta relacionado a ausência de realização de exames ultrassonográficos durante a gestação, já que este não é um exame obrigatório da gestação segundo os protocolos do ministério da saúde (BRASIL, 2009),

No que diz respeito as orientações a cerca do aleitamento materno no período gestacional, $84 \%$ das mães informaram ter recebido algum tipo de orientação, sendo o profissional enfermeiro (52\%) o que mais orientou e o tipo de orientação mais repassada foi a cerca da importância da prática do aleitamento materno (84\%). Este dado reforça que o enfermeiro tem papel fundamental na orientação do aleitamento materno, levandose em consideração seu papel no acompanhamento do pré-natal.

No tocante a utilização do leite materno no período hospitalar $96 \%$ das crianças, tiveram contato com este tipo de leite associado ou não ao leite artificial. Os resultados deste estudo confirmam os evidenciados na literatura já que diversos autores enfatizam o incentivo ao aleitamento natural como orientação básica (SILVA EB e FÚRIA CLB et al., 2005) e visto que apenas $4 \%$ das crianças utilizaram outro leite que não o materno durante seu tempo de permanência hospitalar. Mendes KRR e Lopes VLGS (2006) ressalta que a orientação básica a dar-se para a mãe de um recém-nascido deve enfatizar que o aleitamento materno natural é possível em crianças com fissuras, no entanto a técnica a ser empregada irá depender da complexidade da fissura e condições da criança. Desta forma, entende-se que o suporte profissional durante o período intra-hospitalar, bem como após a alta, é garantia de menores dificuldades na manutenção do aleitamento, seja ele materno ou artificial (CARRARO DF et al., 2011).

As mães afirmaram que entre as crianças com fissuras labiopalatinas, $60 \%$ delas tiveram dificuldades com a pega da criança. Dessa forma considerando que esse é um importante fator causal no déficit ponderal e de crescimento dessas crianças, pois influencia diretamente no estado nutricional, Carraro DF et al. (2011) ressalta que especialmente nos primeiros anos de vida, a presença de fissuras pode resultar na interrupção precoce do aleitamento materno ou mesmo no fato de essas crianças não serem amamentadas.

Este estudo também demonstrou que as crianças que possuíam fissura pré-forame incisivo tiveram uma pega mais efetiva ao seio, o que está de acordo com a literatura vigente e que demonstra ainda que as 
crianças que apresentam maior tempo de aleitamento são as com fissura isolada de lábio (pré-forame) visto que nas fissuras pós e transforame incisivo ocorrem maiores problemas em relação ao aleitamento materno, devido ao comprometimento de uma grande porção do palato havendo uma taxa inadequada de fluxo de leite (CARRARO et al., 2011; SILVA et al. 2005).

Nas fissuras pré-forame incisivo, os recém-nascidos (RN) afetados apresentam menores dificuldades alimentares, quando comparado àqueles com a fenda pós forame incisivo. Nos primeiros a integridade do palato colabora com a manutenção da pressão negativa intraoral e permite ao bebê um melhor padrão de sucção. Entretanto as crianças com fissuras pós-forame incisivo podem apresentar dificuldade de compressão do bico, levando a escape do alimento por não haver adequado ajuste da boca da criança ao bico o que acarretará uma sucção ineficiente e dificultará o processo de alimentação (BRANCO; CARDOSO, 2013).

A mamadeira foi o método mais utilizado pelas mães (60\%) no ambiente domiciliar, e este segundo a literatura, poderá ser utilizado quando não se consegue aleitamento materno através da sucção no peito da mãe. Nestes casos a orientação é ordenhar o leite e oferece-lo a criança em mamadeira, e caso não seja possível deve-se oferecer outro leite, também em mamadeira. Alguns cuidados devem ser tomados quando se escolhe este método alternativo de amamentação tais como escolher o bico adequado considerando alguns fatores como o comprimento, a flexibilidade, o tamanho do furo e a posição adotada na cavidade oral. Os bicos devem ser flexíveis o suficiente para permitirem fácil adaptação na boca da criança e o furo deve estar voltado para cima, ou seja, de encontro ao palato para permitir um fluxo adequado de leite (CARRARO, 2011; MENDES et al., 2009).

Trinta e seis por cento (36\%) das mães relataram a posição sentada como a melhor para amamentar e $24 \%$ essa mesma posição para a criança mamar. Dados similares são encontrados na literatura e estes sugerem deixar o lactente o mais ereto possível durante a mamada, para evitar que os alimentos penetrem na cavidade nasal e para que $o$ ar deglutido possa ser expelido durante a alimentação. A postura adequada também evita que o leite penetre na tuba auditiva, evitando a ocorrência das otites entre as crianças que apresentam o diagnóstico de fissuras labiopalatinas (ALTMANN, 1997; MONLLEÓ, 2014).

Segundo Branco e Cardoso (2013) os bebês portadores de FLP devem ser posicionados semieretos, de frente para o corpo da mãe, com a cabeça inclinada e com a boca direcionada de frente para o mamilo facilitando a pega. Nesta posição, a ação da gravidade permite que o mamilo e a aréola do seio penetrem com mais facilidade dentro da boca do bebê, proporcionando maior vedação da fenda, promovendo assim um melhor escoamento do alimento para a orofaringe e esôfago e diminuindo a possibilidade de refluxo do leite pelo nariz e penetração na tuba auditiva.

\section{CONCLUSÃO}

Pode-se inferir que existe um mau aconselhamento, ou desorientação ou ainda um desconhecimento dos profissionais da área da saúde dos hospitais com relação ao posicionamento correto da criança com fissura visto que a maioria das crianças não teve uma pega efetiva ao seio $(60 \%)$ e, que a maioria das mães relatou não existir uma melhor posição para amamentar e nem para a criança mamar. Desse modo, a própria orientação dos profissionais da saúde, ou a falta dela, podem influenciar negativamente o processo e contribuir para o desmame precoce. Frente ao exposto, percebe-se ainda a necessidade de estudos que aprofundem na causa do desconhecimento da presença da fissura antes do nascimento entre as mães, uma vez que o diagnóstico precoce pode melhorar a aceitação familiar, reduzir o choque frente a anomalia no momento do parto e auxiliar os profissionais de saúde, principalmente o enfermeiro que realiza o pré-natal, a fornecer orientações corretas e especificas pertinentes as técnicas alimentares do bebê fissurado, visto que o ganho de peso é um dos fatores analisados para indicar o tratamento cirúrgico. 


\section{REFERÊNCIAS}

1. ALTMANN EBC et al. Fissuras labiopalatinas. In: PACHI, P.R. Aspectos pediátricos. Carapicuiba: Pró-fono Departamento Editoral, 1997; 367-403 p.

2. BARONEZA JE et al. Dados epidemiológicos de portadores de fissuras labiopalatinas de uma instituição especializada de Londrina, Estado do Paraná. Acta Scientiarum Health Sciences, 2005; 27(1): 31-5.

3. BRANCO LL, CARDOSO MC. Alimentação no recém-nascido em fissuras labiopalatinas. Universitas: Ciências da saúde. Brasília, 2013; 11(1): 57-70.

4. BRASIL. Ministério da Saúde. Saúde da Criança: nutrição alimentar (aleitamento materno e alimentação complementar). Brasilia: Caderno de atenção básica, 2009; n 23.

5. CARRARO DF et al. Fissuras labiopalatinas e nutrição. Revista HCPA. Porto Alegre, 2011; 31(4): 456-463.

6. CARVALHO LRRA. Avaliação das condições bucais de portadores de fissuras lábio palatinas atendidos em serviço de referência no Piauí. Dissertação de mestrado. Universidade Federal do Piaui. Teresina, 2010; 147 p.

7. CYMROT $\mathrm{M}$ et al. Prevalência dos tipos de fissura em pacientes com fissuras labiopalatinas atendidos em um Hospital Pediátrico do Nordeste brasileiro. Rev Bras Cir Plast, 2010; 25(4): 648-51.

8. DI NINNO CQMS et al. Levantamento epidemiológico dos pacientes portadores de fissura de lábio e/ou palato de um centro especializado de Belo Horizonte. Rev Cefac., 2011; 13(6):1002-1008.

9. FIERRO CM, SALAZAR ES et al. Orientación inicial, calidad de consejería y forma de alimentación en niños fissurados. Odontoestomatología, Concepción, 2013; 15(21): 145-7.

10. FREITAS e SILVA DS et al. Estudo descritivo de fissuras labiopalatinas relacionadas a fatores individuais, sistêmicos e sociais. RGO., 2008; 56(4): 387-91.

11. FREITAS AB et al. Fissuras labiopalatinas: estudo sobre a população assistida por um serviço de referência no estado de Minas Gerais. Arquivo em Odontologia , 2009; 45(2):107-12.

12. FREITAS JAS et al. Rehabilitative treatment of cleft lip and palate experience of the Hospital of Rehabilitation of Craniofacial Anomalies/USP- Part 1: overall aspects. J Appl Oral Science, 2011; 12(1): 9-15.

13. FREITAS MCA, BATISTA TS et al. Revista UNINGÁ. Maringá, 2013; 1(37): 13-22.

14. GARDENAL $M$ et al. Prevalência de fissuras orofaciais diagnosticadas em um serviço de referência em casos residentes no estado de Mato Grosso do Sul. Arq. Int. Otorrinolaringol, 2011; 15(2): 133-41.

15. MENDES KRR et al. Amamentação da criança portadora de fissura labiopalatina. Trabalho de Conclusão de Curso. Universidade Vale do Rio Doce. Governador Valadares, 2009; 60p.

16. MENDES LGA, LOPES VLGS. Fenda de lábio e ou palato: recursos para alimentação antes da correção cirúrgica. Rev Cien Médica, Campinas, 2006; 15(5): 437-448.

17. MONLLEÓ IL, MENDES LGA, LOPES VLGS et al. Manual de cuidados de saúde e alimentação da criança com fenda oral. Projeto crânio-face Brasil. Campinas, 2014; 22(5): 913-921.

18. SILVA EB, FÚRIA CLB et al. Aleitamento materno em recém nascidos portadores de fissura labiopalatina: dificuldades e métodos utilizados. Rev CEFAC, São Paulo, 2005; 7(1): 13-20.

19. SILVA RCC, CARMO HA, NETO FRGX et al. Perfil dos casos de fissura labiopalatal atendidos em um hospital de ensino do norte do Ceará, Brasil. Caderno Escola de Saúde Pública do Ceará. Ceará, 2013; 7(2): 19-27.

20. SPINA V et al. Classificação das fissuras lábiopalatais: sugestão de modificação. Revista do Hospital das Clínicas da Faculdade de Medicina da Universidade de São Paulo. São Paulo, 1972; 27(1): 5-6.

21. THOMÉ S. Estudo da prática do aleitamento materno em crianças portadoras de malformações congênita de lábio e/ou de palato. Dissertação (Mestrado). Escola de Enfermagem de Ribeirão Preto, Universidade de São Paulo. Ribeirão Preto, 1990; 244p.

22. WEHBY GL, CASSELL CH. The impact of orofacial clefts on quality of life and healthcare use and costs. Oral Diseases, 2010; 16(1): 3-10. 